\title{
Assessment of the Anti-anginal Effect of Tetramethylpyrazine Using Vasopressin-Induced Angina Model Rats
}

\author{
Xin Cao, ${ }^{a}$ Yuji Nakamura, ${ }^{a}$ Takeshi Wada, ${ }^{a}$ Takuya Kishie, ${ }^{a}$ Azjargal Enkhsaikhan, ${ }^{a}$ \\ Hiroko Izumi-Nakaseko, ${ }^{a}$ Kentaro Ando, ${ }^{a}$ Bingmei Zhu, ${ }^{b}$ Bin Xu, ${ }^{b}$ and Atsushi Sugiyama ${ }^{*, a, c}$ \\ ${ }^{a}$ Department of Pharmacology, Faculty of Medicine, Toho University; 5-21-16 Omori-nishi, Ota-ku, Tokyo 143 \\ 8540, Japan: ${ }^{b}$ Key laboratory of Acupuncture and Medicine Research of Ministry Education, Nanjing University of \\ Chinese Medicine; 138 Xianlin Ave, Qixia, Nanjing, Jiangsu, China: and ${ }^{c}$ Yamanashi Research Center of Clinical \\ Pharmacology; 73-5 Hatta, Isawa-cho, Fuefuki, Yamanashi 406-0023, Japan.
}

Received January 20, 2016; accepted April 7, 2016

Intravenous tetramethylpyrazine has been widely used in China as a complementary and/or alternative medicine to treat patients with ischemic heart disease. We assessed the anti-anginal effect of tetramethylpyrazine $(10 \mathrm{mg} / \mathrm{kg}$, intravenously (i.v.), $n=6)$ in comparison with that of its vehicle, saline $(1 \mathrm{~mL} / \mathrm{kg}$, i.v., $n=6)$, using vasopressin-induced angina model rats. First, Donryu rats were anesthetized with pentobarbital sodium $(60 \mathrm{mg} / \mathrm{kg}$, intraperitoneally (i.p.)), and the surface lead I electrocardiogram was continuously monitored. Administration of vasopressin $(0.5 \mathrm{IU} / \mathrm{kg}$, i.v.) to the rats depressed the S-wave level of the electrocardiogram, indicating the onset of subendocardial ischemia. However, pretreatment with tetramethylpyrazine suppressed the vasopressin-induced depression of the S-wave level, which was not observed following pretreatment with its vehicle alone (saline), suggesting that tetramethylpyrazine ameliorated the vasopressininduced subendocardial ischemia in vivo. These results may provide experimental evidence for the empirically known clinical efficacy of tetramethylpyrazine against ischemic heart disease, and could provide clues to better understanding its in vivo mechanism of action.

Key words tetramethylpyrazine; vasospastic angina; ST-segment depression

While $\beta$-blockers, $\mathrm{Ca}^{2+}$ antagonists and/or nitrates have been used for the treatment of patients with angina pectoris according to therapeutic guidances of western medicine, ${ }^{1)}$ tetramethylpyrazine (TMP), an extract of the herb Ligusticum wallichii FRANCH. (chuanxiong), has been widely applied for the treatment of ischemic heart disease as a complementary and/or alternative medicine in China. ${ }^{2)}$ Its molecular and cellular levels of mechanisms of actions have been reported to include $\mathrm{Ca}^{2+}$ antagonism, ${ }^{3)}$ activation of $\mathrm{Ca}^{2+}$-activated potassium current, ${ }^{4)}$ stimulation of cyclic adenosine monophosphate production $^{5)}$ and enhancement of endothelium-dependent nitric oxide synthesis. ${ }^{6}$ Moreover, pretreatment of tetramethylpyrazine was shown to ameliorate a vasoconstrictor terlipressin-induced decrease of cardiac index in portal hypertensive rats. ${ }^{7)}$ However, in vivo experimental evidence for empirically known clinical efficacy of tetramethylpyrazine is still limited. In the present study, we assessed the effects of tetramethylpyrazine on the vasopressin-induced angina model of rats, which has been shown to be useful and reliable in evaluating the anti-spastic effect of a drug in vivo. ${ }^{8)}$

\section{MATERIALS AND METHODS}

Experiments were performed by using 12 male Donryu rats, weighing approximately 200-300g (Japan SLC, Inc., Shizuoka, Japan). Animals were kept at $23 \pm 1^{\circ} \mathrm{C}$ under a $12-\mathrm{h}$ light-dark cycle, where food and water were available. All experiments were approved by the Animal Research Committee for Animal Experimentation of Toho University (No. 15-53-251) and performed in accordance with the Guidelines for the Care and Use of Laboratory Animal of Toho University.
Cardiovascular Variables The rats were divided into two groups: tetramethylpyrazine group $(n=6)$ and vehicle group $(n=6)$. The animals were anesthetized with pentobarbital sodium $(60 \mathrm{mg} / \mathrm{kg}$, intraperitoneally (i.p.)). The right femoral vein and artery were cannulated for drug administration and for measuring blood pressure, respectively. Additional pentobarbital sodium $(10-20 \mathrm{mg} / \mathrm{kg}$, intravenously (i.v.)) was injected through the femoral vein to maintain an adequate depth of anesthesia, which was judged by jaw muscle tone. Body temperature was maintained at $37^{\circ} \mathrm{C}$ with a heating pad. The surface lead I electrocardiogram was obtained from the limb electrodes. The blood pressure and electrocardiogram were continuously monitored with a polygraph system (RM-6000; Nihon Kohden Co., Tokyo, Japan), which were analyzed with a real time full automatic data analysis system (WinVAS3 ver 1.1R24v; Physio-Tech Co., Ltd., Tokyo, Japan).

Experimental Protocol The protocol of the whole experiment was shown in Fig. 1. After the assessment of basal control values $(\mathrm{C})$, vehicle saline $(1 \mathrm{~mL} / \mathrm{kg}$, i.v. $)$ was infused over $1 \mathrm{~min}$. Six minutes after the start of the vehicle administration, vasopressin (VP) $(0.5 \mathrm{IU} / \mathrm{kg}$, i.v.) was infused over $1 \mathrm{~min}$ to induce ischemic attack. More than $30 \mathrm{~min}$ after the start of initial vasopressin administration, tetramethylpyrazine $(10 \mathrm{mg} /$ $\mathrm{kg}$, i.v.) or vehicle $(1 \mathrm{~mL} / \mathrm{kg}$, i.v.) was infused over $1 \mathrm{~min}$. Six minutes after the start of tetramethylpyrazine or vehicle administration, vasopressin $(0.5 \mathrm{IU} / \mathrm{kg}$, i.v. $)$ was infused over $1 \mathrm{~min}$. The dose of tetramethylpyrazine in this study was determined by previous experimental studies ${ }^{2-7)}$ as well as clinical experiences as a traditional Chinese medicine.

Drugs Tetramethylpyrazine was obtained from Medisan Pharmaceuticals Co., Ltd. (Harbin, China). Vasopressin was purchased from Daiichi Sankyo Co., Ltd. (Tokyo, Japan). Tet- 
ramethylpyrazine was dissolved in saline at a concentration of $10 \mathrm{mg} / \mathrm{mL}$. Vasopressin was diluted with saline to a concentration of $0.5 \mathrm{IU} / \mathrm{mL}$. The other drugs used were pentobarbital sodium (Tokyo Chemical Industry Co., Ltd., Tokyo, Japan).

\section{Vehicle Group $(n=6)$}

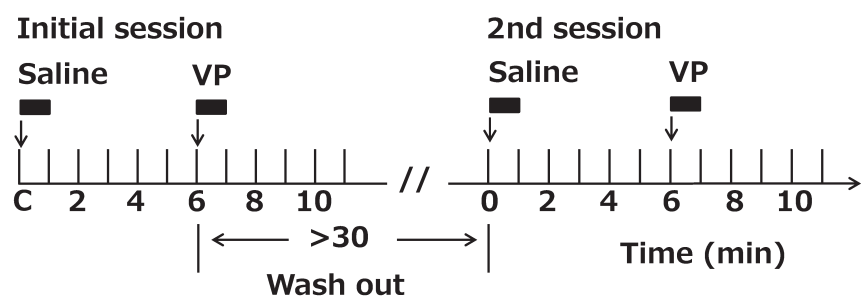

\section{TMP Group $(n=6)$}

\section{Initial session}

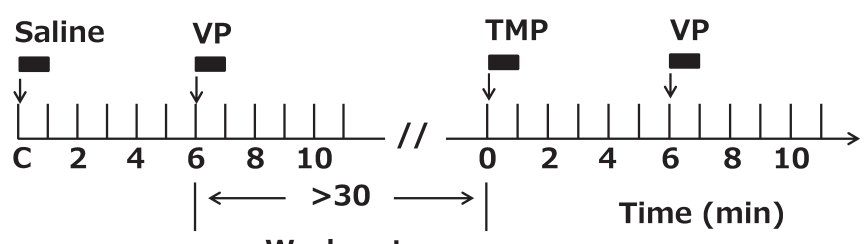

\section{Wash out}

Fig. 1. Schematic Diagram of Experimental Protocol for the Tetramethylpyrazine (TMP) and Vehicle Groups

In the initial session, vehicle $(1 \mathrm{~mL} / \mathrm{kg}$, i.v. $)$ was infused for each group; then, vasopressin (VP; $0.5 \mathrm{IU} / \mathrm{kg}$, i.v.) was administered to induce ischemic attack. In the 2nd session, $>30 \mathrm{~min}$ after the initial VP administration, TMP $(10 \mathrm{mg} / \mathrm{kg}$, i.v. $)$ or the vehicle $(1 \mathrm{~mL} / \mathrm{kg}$, i.v. $)$ was administered; then, VP $(0.5 \mathrm{IU} / \mathrm{kg}$, i.v. $)$ was administered to induce ischemic attack. Each infusion was performed over $1 \mathrm{~min}$ Parameters were assessed every minute for $5 \mathrm{~min}$ after the start of infusion of TMP or the vehicle. Basal control value in the initial session (C).

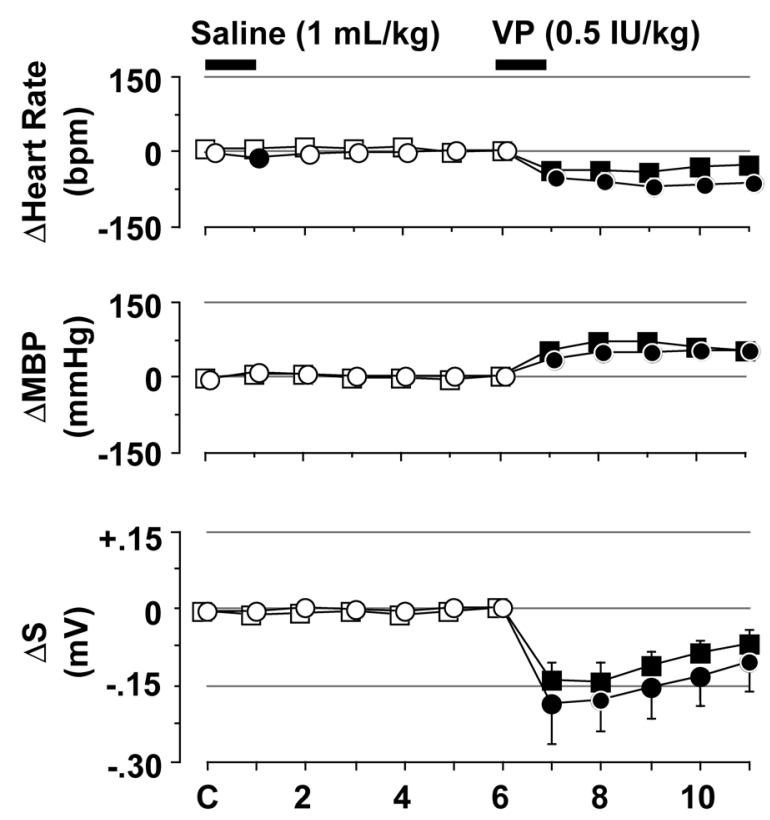

Statistical Analysis The depression of the S-wave level was measured in three consecutive recordings of lead I electrocardiogram, which was used for a marker to estimate the severity of the subendocardial ischemia. ${ }^{8)}$ The effects of tetramenthylpyrazine (TMP) and vehicle on the vasopressin (VP)-induced changes in the heart rate from that just before vasopressin administration $(\Delta \mathrm{HR})$, mean blood pressure from that just before vasopressin administration $(\triangle \mathrm{MBP})$ and $\mathrm{S}$ wave level from that just before vasopressin administration $(\Delta \mathrm{S})$ were calculated in order to precisely analyze the impact of vasopressin on each cardiovascular variable. Data are presented as the mean \pm standard error of the mean (S.E.M.). The statistical significances within a parameter were evaluated by repeated-measures ANOVA followed by Contrasts as a post$h o c$ test for mean values comparison. A $p$ value $<0.05$ was considered to be statistically significant.

\section{RESULTS}

Effects on the Heart Rate Basal control value (C) was $415 \pm 25 \mathrm{bpm}$ in the tetramethlpyradine group $(n=6)$, and $337 \pm 11 \mathrm{bpm}$ in the vehicle group $(n=6)$. The time courses of changes in the heart rate from that just before the vasopressin administration $(\triangle \mathrm{HR})$ are summarized in Fig. 2. There was no significant difference in the control value of $\Delta H R$. In the initial session, administration of the vehicle hardly affected the heart rate in the tetramethylpyrazine group, but it decreased the heart rate at $1 \mathrm{~min}$ after the start of infusion in the vehicle group compared with that just before the vasopressin administration. Additional administration of vasopressin decreased the heart rate for $1-5 \mathrm{~min}$ in both groups. In the second session, administration of tetramethylpyrazine increased the heart rate at $1 \mathrm{~min}$ after the start of infusion in the tetramethylpyrazine

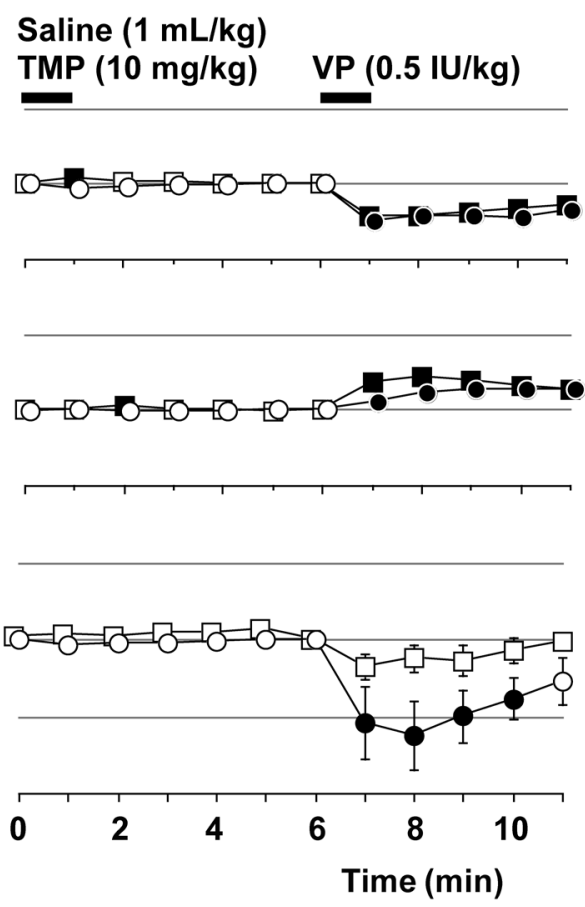

Fig. 2. Summary of the Effects of Tetramenthylpyrazine (TMP) and Vehicle on the Vasopressin (VP)-Induced Changes in the Heart Rate from That Just before Vasopressin Administration $(\Delta \mathrm{HR})$, Mean Blood Pressure from That Just before Vasopressin Administration $(\Delta \mathrm{MBP})$ and S-Wave Level from That Just before Vasopressin Administration $(\Delta \mathrm{S})(n=6$ for Each Group)

Data are presented as the mean \pm S.E.M. The results of the tetramethylpyrazine group are indicated as squares, whereas those of the vehicle group are shown as circles for comparison. Closed symbols represent statistically significant differences from the value that just before the vasopressin administration by $p<0.05$. 


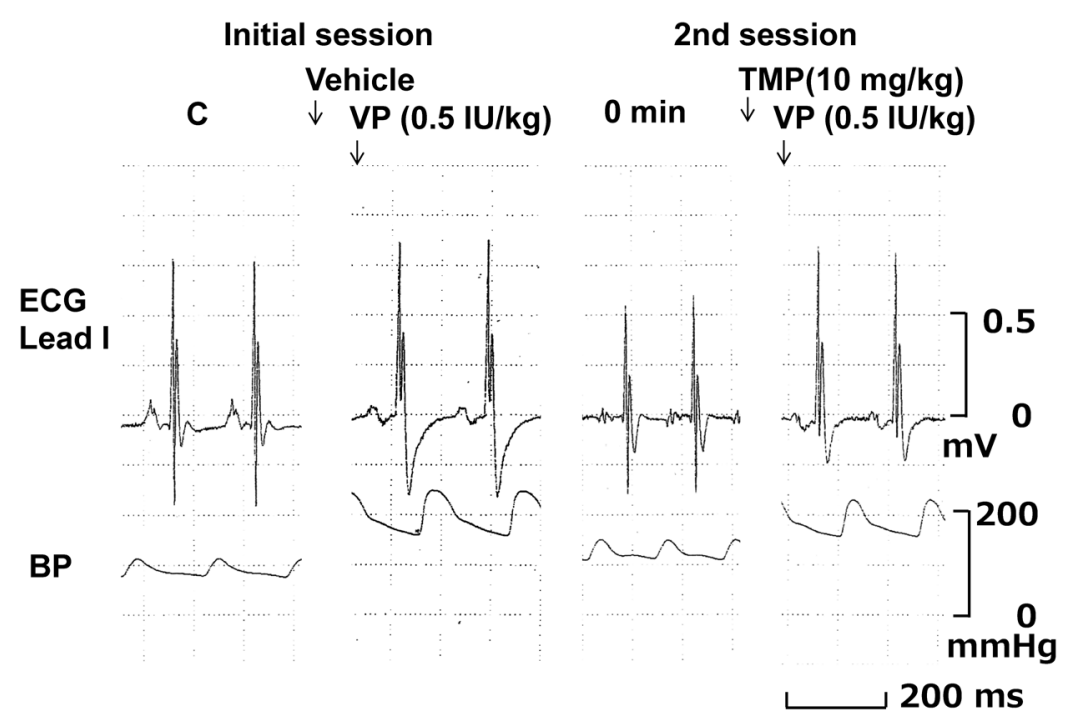

Fig. 3. Typical Tracings Showing the Effects of Tetramethylpyrazine (TMP) on the Vasopressin (VP)-Induced Change in the Electrocardiogram (ECG) and Blood Pressure (BP) in Donryu Rats

Note that the VP-induced depression of the S-wave level was inhibited by pretreatment of TMP. Basal control value in the initial session (C).

group compared with that just before the vasopressin administration, whereas administration of the vehicle hardly affected the heart rate in the vehicle group. Additional administration of vasopressin decreased the heart rate in both groups.

Effects on the Blood Pressure Basal control value (C) was $92 \pm 3 \mathrm{mmHg}$ in the tetramethylpyrazine group $(n=6)$ and $112 \pm 9 \mathrm{mmHg}$ in the vehicle group $(n=6)$. The time courses of changes in the mean blood pressure from that just before the vasopressin administration $(\triangle \mathrm{MBP})$ are summarized in Fig. 2. Typical tracings showing the effects of tetramethylpyrazine on the vasopressin-induced changes in the blood pressure are depicted in Fig. 3. There was no significant difference in the control value of $\triangle \mathrm{MBP}$. In the initial session, administration of vehicle hardly affected the mean blood pressure in both groups. Additional administration of vasopressin increased the mean blood pressure for $1-5 \mathrm{~min}$ after the start of infusion in both groups. In the second session, administration of tetramethylpyrazine increased the mean blood pressure at 2 min after the start of infusion in the tetramethylpyrazine group compared with that just before the vasopressin administration, whereas administration of vehicle hardly affected the mean blood pressure in the vehicle group. Additional administration of vasopressin increased the mean blood pressure for $1-5 \mathrm{~min}$ after the start of infusion in both groups.

Anti-anginal Effects The time courses of changes in the S-wave level from that just before the vasopressin administration $(\Delta S)$ are summarized in Fig. 2. Typical tracings of the effects of tetramethylpyrazine on the vasopressin-induced changes in electrocardiogram are depicted in Fig. 3. Basal control value (C) was $-0.007 \pm 0.010 \mathrm{mV}$ in the tetramethylpyrazine group $(n=6)$, and $-0.005 \pm 0.009 \mathrm{mV}$ in the vehicle group $(n=6)$. There was no significant difference in the control value of $\Delta \mathrm{S}$. In the initial session, administration of the vehicle hardly affected the $\Delta S$-wave level in both groups. Additional administration of vasopressin depressed the $\Delta S$-wave level for $1-5 \mathrm{~min}$ after the start of infusion in both groups. In the second session, administration of tetramethylpyrazine or vehicle hardly affected the $\Delta \mathrm{S}$-wave level in both groups. Additional administration of vasopression hardly affected the
$\Delta \mathrm{S}$-wave level in the tetramethylpyrazine group, whereas it depressed the $\Delta \mathrm{S}$-wave level for $1-4 \mathrm{~min}$ after the start of infusion in the vehicle group.

\section{DISCUSSION}

In the present study, we compared in vivo anti-anginal effect of tetramethylprazine with that of its vehicle by using a well-established vasospastic angina model of rats. ${ }^{7)}$ Intracoronary administration of vasopressin has been shown to constrict small coronary arteries, causing subendocardial ischemia ${ }^{9)}$ and $\mathrm{Ca}^{2+}$ channel blocker is known to possess a potent coronary vasodilator effect, which has been widely used for patients with vasospastic angina. ${ }^{1)}$ Tetramethylpyrazine significantly inhibited the vasopressin-induced subendocardial ischemia, which was not observed by the vehicle, indicating that tetramethylpyrazine may possess an anti-ischemic effect. Similar results were reported previously. Intravenous administration of $2-15 \mathrm{mg} / \mathrm{kg}$ tetramethylpyrazine was reported to increase the coronary blood flow from 37 to $74 \mathrm{~mL} / \mathrm{min}$ but decrease the mean coronary vascular resistance from 1770 to $700 \mathrm{dyn} \cdot \mathrm{s} \cdot \mathrm{cm}^{-3}$ in a dose-related manner in anesthetized dogs. ${ }^{10)}$ Moreover, pretreatment of tetramethylpyrazine $(80 \mathrm{mg} /$ $\mathrm{kg}$, i.v.) suppressed the endothelin-1-induced coronary vasoconstriction in anesthetized dogs. ${ }^{11)}$ Several potential mechanisms that may explain the coronary vasodilator effects of tetramethylpyrazine have been proposed. Tetramethylpyrazine has been shown to stimulate $\mathrm{Ca}^{2+}$-activated potassium current, ${ }^{4)}$ cyclic adenosine monophosphate production, ${ }^{5)}$ and the endothelial nitric oxide synthase ${ }^{6)}$ in addition to $\mathrm{Ca}^{2+}$ channel blockade. ${ }^{3)}$ While these previous knowledge regarding the coronary vasodilator action of tetramethylpyrazine may partly explain its currently observed anti-anginal effect, its precise mechanisms need to be elucidated.

Vasopressin in doses of $0.12,0.4,1.2$ and $4 \mathrm{mU} / \mathrm{kg} / \mathrm{min}$ has been reported to increase the left ventricular end-diastolic pressure in a dose-related manner in the pentobarbital sodiumanesthetized dogs. ${ }^{12)}$ Since the elevation of the preload can increase the ventricular wall stress, resulting in aggravation of 
subendocardial ischemia, ${ }^{13)}$ the effect of a drug on the preload to the left ventricle may have some potential to affect its antianginal effects. It has been shown that tetramethylpyrazine could significantly decrease the left ventricular end-diastolic pressure in isoproterenol-induced heart failure in rats. ${ }^{14)}$ Thus, the pretreatment of tetramethylpyrazine might have ameliorated the vasopressin-induced increase of the left ventricular end-diastolic pressure, which could also contribute to the antianginal effect.

The currently observed results may provide an in vivo experimental evidence for empirically known clinical efficacy of tetramethylpyrazine against ischemic heart disease, and suggest that tetramethylpyrazine could be applied for patients with vasospastic angina.

Acknowledgments This study was supported in part by the Project Research Grant of Toho University School of Medicine (No. 27-20), JSPS KAKENHI (\#25460344), MEXT KAKENHI (\#S1101016) and AMED Grant (\#AS2116907E). We thank Ms. Misako Nakatani and Mrs. Yuri Ichikawa for their technical assistance.

Conflict of Interest The authors declare no conflict of interest.

\section{REFERENCES}

1) Hung MJ, Hu P, Hung MY. Coronary artery spasm: review and update. Int. J. Med. Sci., 11, 1161-1171 (2014).

2) Chen H, Li G, Zhan P, Liu X. Ligustrazine derivatives. Part 5: design, synthesis and biological evaluation of novel ligustrazinyloxycinnamic acid derivatives as potent cardiovascular agents. Eur. J. Med. Chem., 46, 5609-5615 (2011).

3) Pang PK, Shan JJ, Chiu KW. Tetramethylpyrazine, a calcium antagonist. Planta Med., 62, 431-435 (1996).

4) Yang YY, Yang Y, Zeng XR, Liu ZF, Cai F, Li ML, Zhou W, Pei J. Effects of tetramethylpyrazine on large-conductance $\mathrm{Ca}^{2+}$-activated potassium channels in porcine coronary artery smooth muscle cells. Sheng Li Xue Bao, 58, 83-89 (2006).

5) Wu CC, Chiou WF, Yen MH. A possible mechanism of action of tetramethylpyrazine on vascular smooth muscle in rat aorta. Eur. J. Pharmacol., 169, 189-195 (1989).

6) Lv L, Jiang SS, Xu J, Gong JB, Cheng Y. Protective effect of ligustrazine against myocardial ischaemia-reperfusion in rats: the role of endothelial nitric oxide synthase. Clin. Exp. Pharmacol. Physiol., 39, 20-27 (2012).

7) Chang FC, Huang YT, Lin HC, Hong CY, Lin JG, Chen KJ. Beneficial effects of combined terlipressin and tetramethylpyrazine administration on portal hypertensive rats. Can. J. Physiol. Pharmacol., 77, 618-624 (1999).

8) Yao K, Shirakura S. Combined effects of benidipine and diltiazem in a rat model of experimental angina. J. Pharmacol. Sci., 95 , 394-397 (2004).

9) Maturi MF, Martin SE, Markle D, Maxwell M, Burruss CR, Speir E, Greene R, Ro YM, Vitale D, Green MV, Goldstein SR, Bacharach SL, Patterson RE. Coronary vasoconstriction induced by vasopressin. Production of myocardial ischemia in dogs by constriction of nondiseased small vessels. Circulation, 83, 2111-2121 (1991).

10) Dai XZ, Bache RJ. Coronary and systemic hemodynamic effects of tetramethylpyrazine in the dog. J. Cardiovasc. Pharmacol., 7, 841-849 (1985)

11) Zeng Z, Zhu W, Zhou X, Jin Z, Liu H, Chen X, Pan J, Demura $\mathrm{H}$, Naruse M, Shi Y. Tetramethylpyrazine, a Chinese drug, blocks coronary vasoconstriction by endothelin-1 and decreases plasma endothelin-1 levels in experimental animals. J. Cardiovasc. Pharmacol., 31 (Suppl. 1), S313-S316 (1998).

12) Yatsu T, Kusayama T, Tomura Y, Arai Y, Aoki M, Tahara A, Wada $\mathrm{K}$, Tsukada J. Effect of conivaptan, a combined vasopressin $\mathrm{V}_{1 \mathrm{a}}$ and $\mathrm{V}_{2}$ receptor antagonist, on vasopressin-induced cardiac and haemodynamic changes in anaesthetized dogs. Pharmacol. Res., 46, 375-381 (2002)

13) Dunn RB, Griggs DM Jr. Ventricular filling pressure as a determinant of coronary blood flow during ischemia. Am. J. Physiol., 244, H429-H436 (1983).

14) Guo L, Wang A, Sun Y, Xu C. Evaluation of antioxidant and immunity function of tetramethylpyrazine phosphate tablets in vivo. Molecules, 17, 5412-5421 (2012). 